\title{
Delivery Demand Peak Leveling Based on Customer's Acceptance Capability Analysis
}

\author{
Yuki MATSUDA and Kenji TANAKA \\ The University of Tokyo, Graduate School of Engineering
}

\begin{abstract}
As large scale E-commerace platforms rise, the delivery demand peaks are becoming more remarkable. This is mainly caused by sale promotions and oneday delivery promises. In this field, there is an implicit assumption that re-allocating the deliveries to level delivery peak results in lowering customer utility and thus, the mainstream approach is to make efficient the work plan in given conditions. This paper analyzes real delivery data, and indicates the possibility of delivery reallocation without lowering customer utility, if an transdisciplinary approach of customer analysis and vehicle routing is applied. A delivery allocation system based on this implication was proposed, and a simulation based case study was conducted. Through this, the effect on delivery demand peak leveling without lowering customer utility, was verified.
\end{abstract}

Keywords. Delivery peak leveling, Customer acceptance capability analysis, Realtime delivery capacity analysis, Customer-Logistics capability matching

\section{Introduction}

As large scale E-commerce (EC) platforms rise [1], the delivery demand peaks are becoming more remarkable. This peak is mainly caused by sales promotions and oneday delivery promises. These peaks result in low occupancy rate of deilivery assets and work enviornment detoriation of delivery workers. Due to its large impact on delivery cost and quality, this is one of the major issues for logistic companies.

The main approach taken now, is to take this demand peak as a precondition, and to make the work plan efficient using both strict algorythms and heuristic algorythms [2]. An alternate approach exists of analyzing and smoothing the demand peak through delivery allocaton [3][4][5], but this usually results in lowering customer utility and thus, is not considered much practically.

However, there is a limitation to the existing optimization approach and an alternative approach is needed. Through real data analysis, this paper aims to show the possibility of delivery re-allocation without lowering customer utiliy if an interdiciplinarty approach of customer analysis and logistics analysis is taken.

In Section 2 and Section 3, the possible effectiveness of this approach is shown through analysis of delivery result data of a Japanese logistics company. In Section 4, a delivery allocation system is proposed based on this implicaton. The systems's peak shifting effect is evaluated in Section 5, following with a conclustion in Section 6. 


\section{Customer acceptance capability analysis}

When considering delivery re-allocation, customers tend to be considered as one large persona. An example of this is shown in Figure 1. Although this gives a broad understanding ande model of customer acceptance capability, important information when considering precise delivery allocation is lost in the aggregation process. In this analysis, delivery result data from $2018 / 9 / 23$ to $2020 / 4 / 31$ of a Japanese to-Consumer EC and logistics company is used.

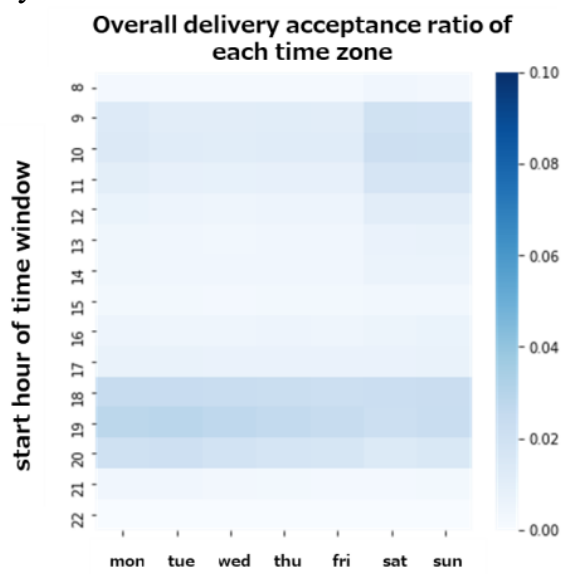

Figure 1. Overall average customer calculated from delivery data.

One information lost is the time zone in which each customer are capable of receiving their deliveries. Figure 2 shows a clustering result of customers using features acceptance hour ratio (8:00 14:00/14:00 18:00/18:00 23:00), and acceptance day of week ratio (weekdays/weekends). Cusomers with more than 20 orders within the data period was used. This shows that although largest cluster is close to the overall average, there are many smaller yet sufficient clusters where customers accept packages at a different time zone.

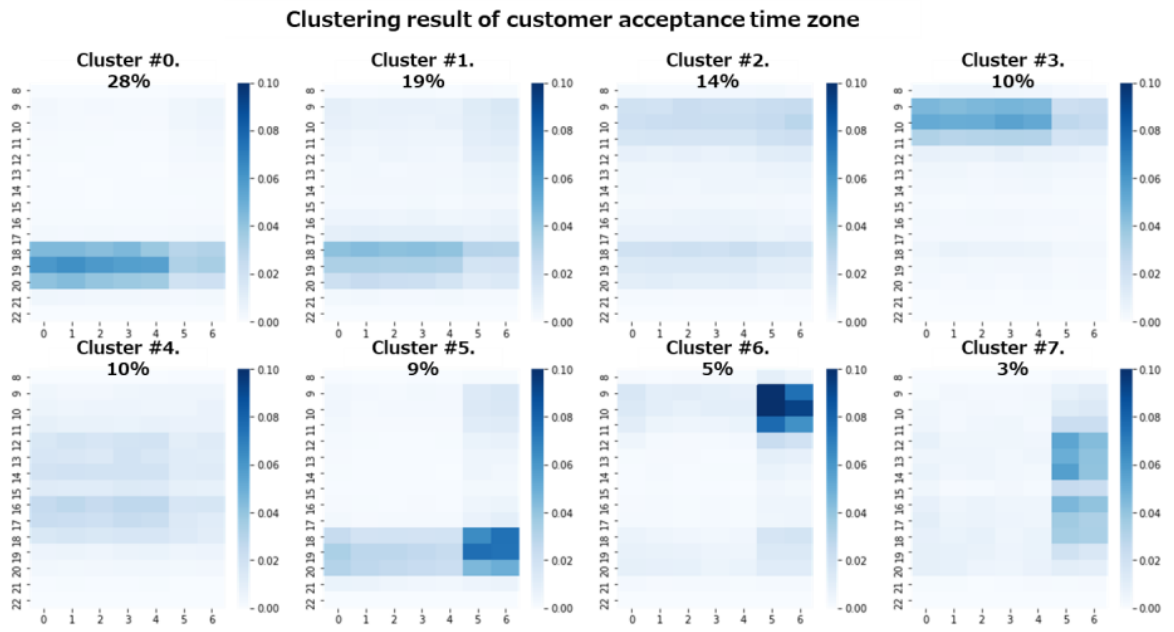

Figure 2. Clustering result of customer acceptance time zone. 
Another information lost is the area size of acceptance of each customer. There are customers who only accept deliveries at a narrow time zones and others who accept them at a wider one. Figure 3 shows the distribution of the area size of acceptance, defined by the number of cells needed to cover $50 \%$ of the customer's acceptance performance. It is shown that the area size varies per customer.

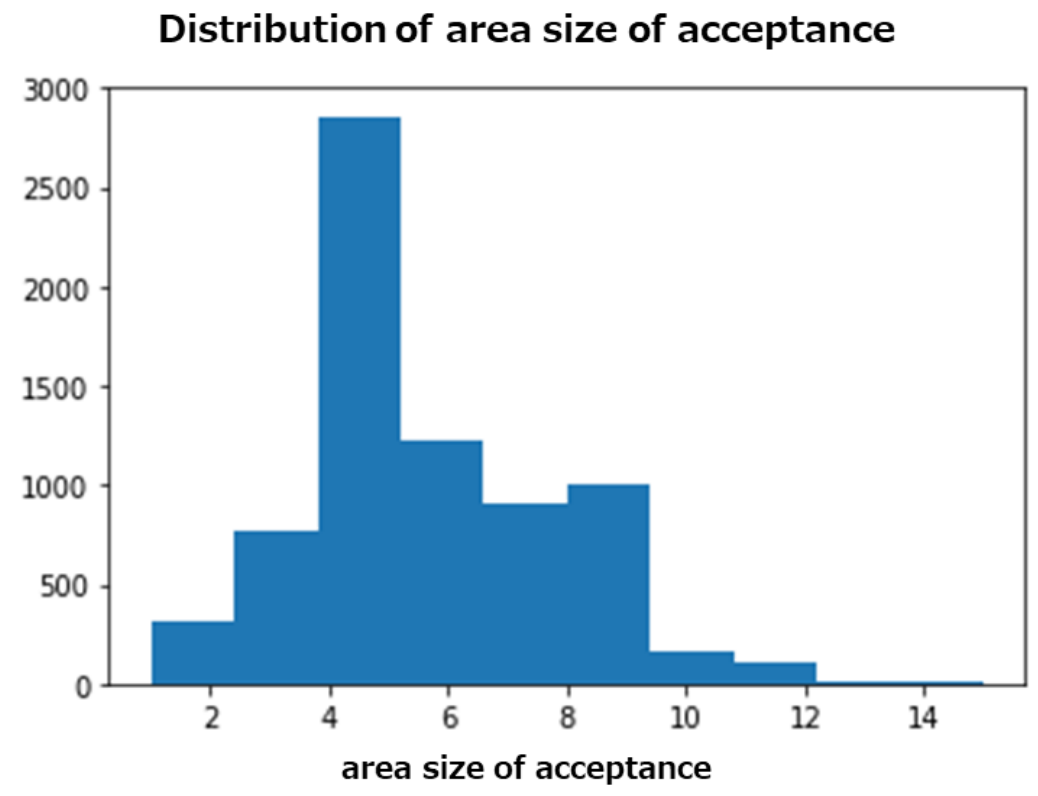

Figure 3. Distribution of area size of accpetance.

From the analysis above, it is shown that the delivery acceptance capability of each customer vaies sufficiently, and that this variance could be used to allocate deliveries to certain customers.

\section{Delivery capability analysis}

To accomplish a viable delivery allocation, in addition to the customer acceptance capability point of view, the delivery side point of view is also needed. Without this, an excess amout of deliveries could be allocated to a certain time zone.

At the present, there is a heuristic static capability limit set for each delivery zone or truck driver at each time zone in order to avoid this overflow. However, this results in a very conservative limit and tends to lower the occupancy rate and also lowers customer utility through delivery refusal at certain time zones

In contrast with the current static capacity approach, a real time delivery capability analysis is proposed. This concept is shown in Figure 4. In this approach, following the conditions shown in Equation 1, an approximate efficient delivery route is consecutively calculated. The conditins in equation 1 assure that order restrictions of time windows are met. Through this approach, the geographical characteristcs of each 
customer could be taken into account and thus, a finer delivery capacity analysis could be achieved.
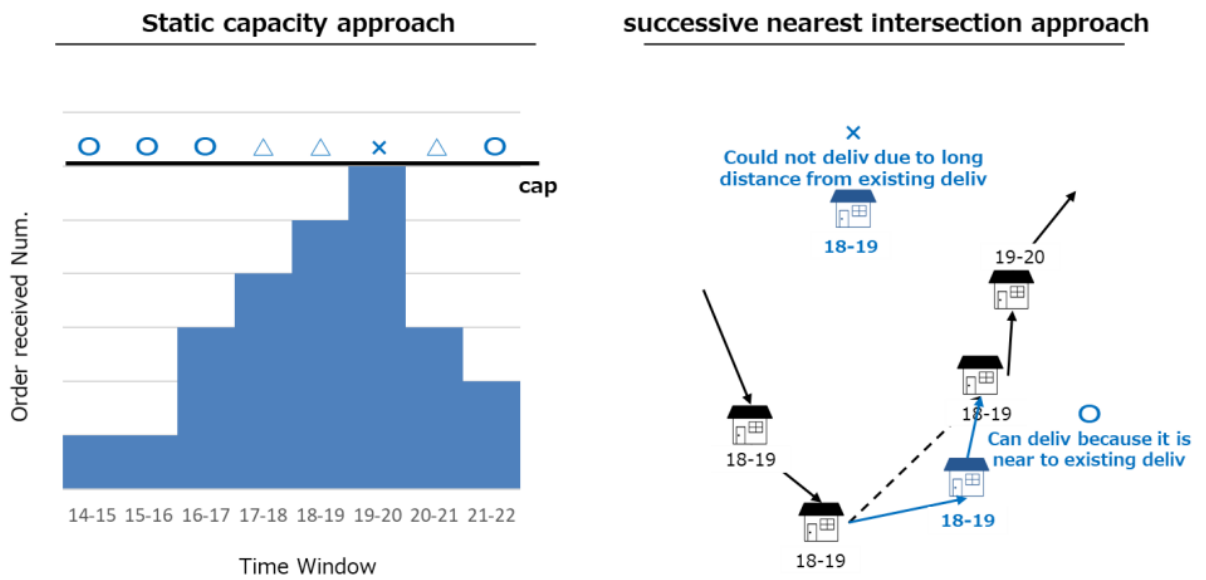

Figure 4. Concept of proposed real time delivery capability analysis.

$\min$

$$
\text { Index }_{i, j}=\text { timeDelta }_{i, n e w C u s t o m e r}+\text { timeDelta }_{\text {newCustomer }, j}-\text { timeDelta }_{i, j}
$$

S.t

$$
\begin{aligned}
& \operatorname{timeWindow}_{\mathrm{f}}(a) \leq \text { timeWindow }_{s}(b) \Leftrightarrow a<b \\
& \text { timeWindow }_{\mathrm{f}}(b) \leq \text { timeWindow }_{\mathrm{s}}(a) \Leftrightarrow b<a
\end{aligned}
$$

$$
\begin{array}{ll}
\text { timeWindow }_{s}(\mathrm{x}) & \text { : time window start of customer } \mathrm{x} \\
\text { timeWindow }_{f}(\mathrm{x}) & \text { : time window end of cusomer } \mathrm{x}
\end{array}
$$

Through simulation, it was shown that this approach could correctly expands the limits of delivery accpetance capacity. Delivery data of a delivery area with fine data was selected and used in this simulation. There is an one-to-one correspondence in delivery are and driver in this data. From the distribution of number of customers visited in a delivery shown in Figure 5, it could be seen that the ordinary capacity is around 15 20 customers throughout the delivery. The outliers happen when there is no other option but to exceed the limits heuristically set, and this results in sudden overwork of drivers. Figure 6 shows the distribution of the number of customers which could be visited in simulation within various order scenarios. The orders are generated according to an aggregated probability of the real delivery data, and delivery parameters such as delivery time off truck are set with margin. Thus, this number is both realistic and viable. 


\section{Distribution of number of customer visited in delivery}

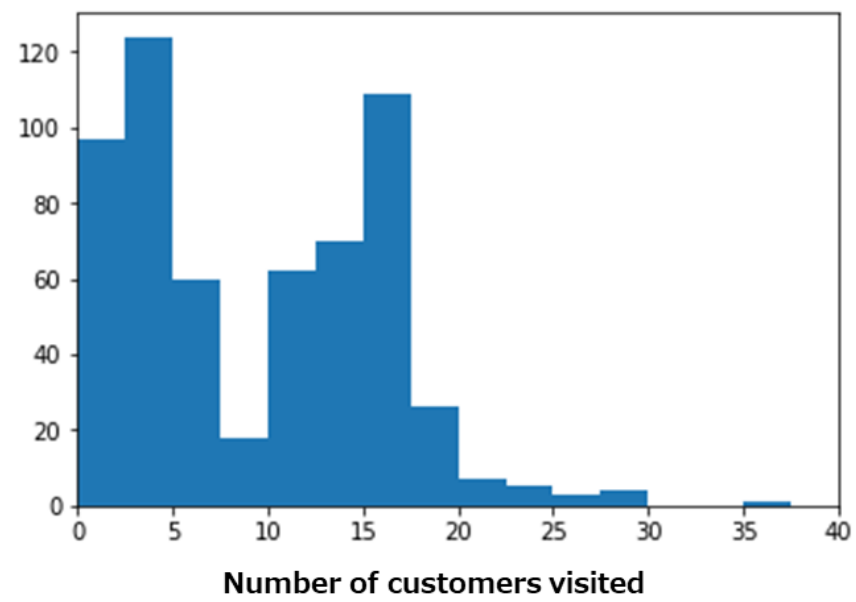

Figure 5. Current distribution of customers visted in delivery.

\section{Distribution of simulation result of number of customer visited in delivery}

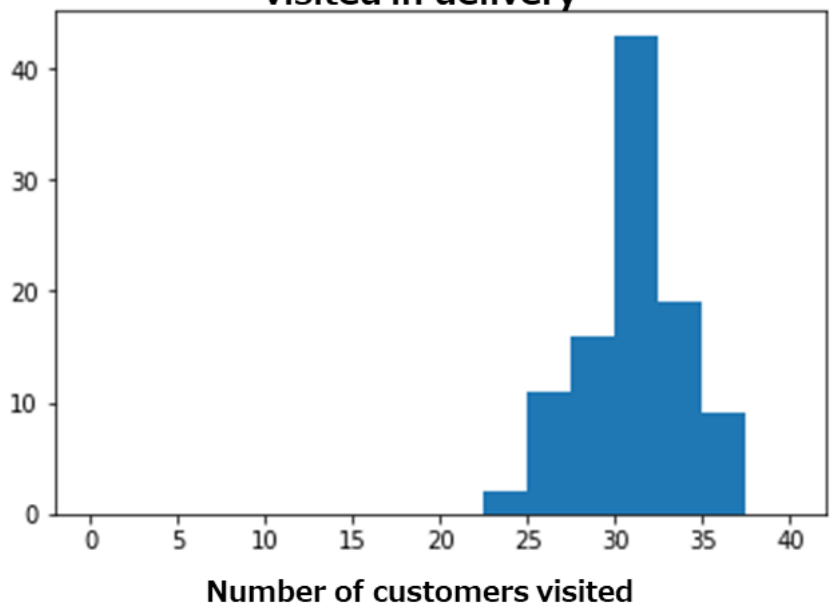

Figure 6. Distribution of number of customers which could be achieved.

From the analysis above, it is shown that delivery capacity could be finer derived through the successive nearest intersection method. This could correctly expand the logistics side capabitliy, and make re-allocation of deliveries more effective.

\section{Proposed System}

Based on the implications of customer acceptance capability analysis in Section 2 and delivery capability analysis in Section 3, a delivery allocation system shown in 
Figure 7 was proposed. In this system, customer acceptance capability is estimated from past delivery results, and realtime develiry capacity for each customer is estimated from the order status. The two estimations are then matched, to derive the delivery timezone.

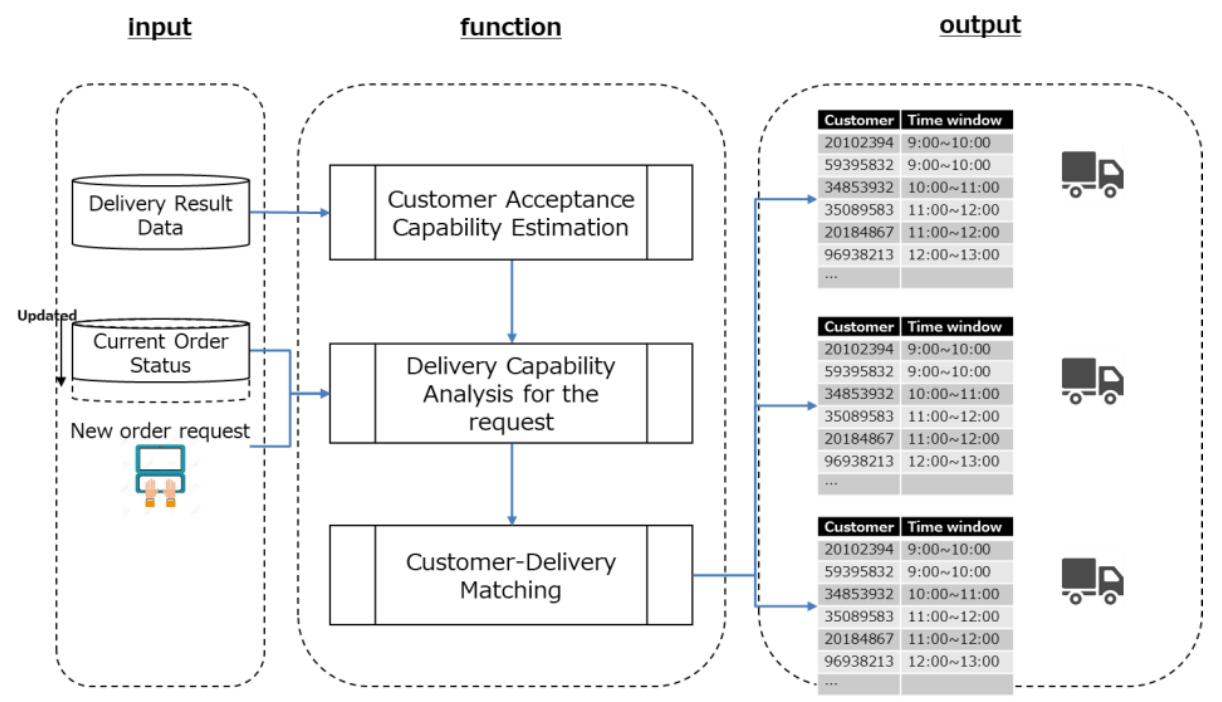

Figure 7. Proposed overall delivery allocation system.

\subsection{Customer acceptance capability estimation}

The delivery results for each cusomer are aggregated to derive the customer acceptance capability. The customers with insufficient ammount of data would be applied with an ordianry one-day delivery.

\subsection{Delivery capacity estimation}

A mix of the confidential static capacity approach, and the successive nearest intersection approach shown in Section 3 is used. The confidential approach is first taken to measure the remaining delivery capacity of each time zone. After this is exceeded, the succsssive nearest intersection approach is applied, and if the customer is judged deliverable, the remaining delivery capacity would be set as 1 for the specific customer.

\subsection{Customer-Delivery matching}

After the customer acceptance capability matrix and delivery capacity matrix is calculated by Sections 4.1 and 4.2, the hour and weekday which minimizes equation 2 is selected as the delivery date and time. This concept is shown in Figure 7. The two matrixes are both normalized so that the sum of numbers add up to one when put into this function. $\beta$ and $\gamma$ are parameters set to express the priority of customer and delivery 


\section{Customer capability}
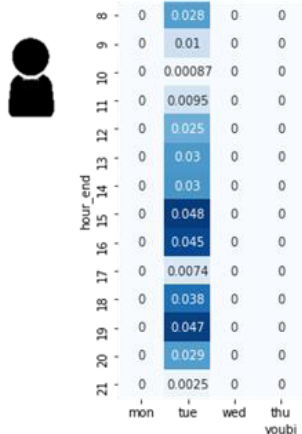

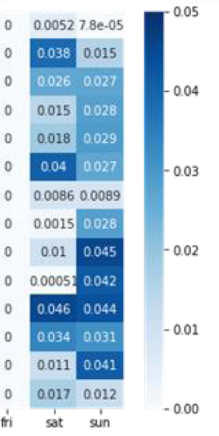

\section{Delivery capability}

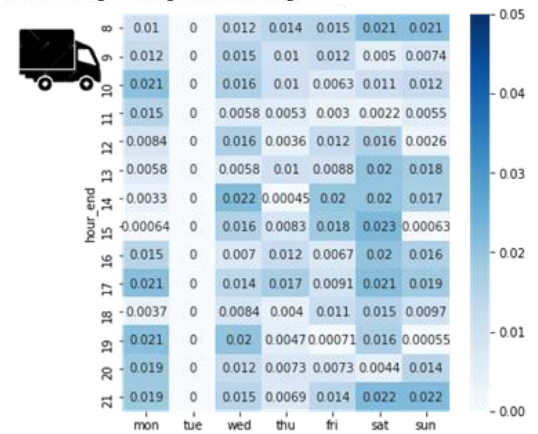

Figure 7. Customer - Delivery matching concept.

$$
\text { matchIndex }_{\text {hour,day }}=\beta * \text { customerCap }_{\text {hour,day }}+\gamma * \text { delivCap } \text { hour }, \text { day }_{\text {, }}
$$

\section{Case study}

Through a case study based on the delivery data mentioned in Section 2, the peak shift effect was validated. The delivery area mentioned in Section 3 was used for the same reasons.

In this case study, a scenario where an excess ammount of orders are given from customers in one single day. The status quo is set as trying to deliver all the orders by one-day delivery. As stated in Section 3 the area is usually delivered by a single driver, but in these occurances, a second, third driver is committed using high cost.

The result of peak shifting is shown in Figure 8. It is shown that when applying the proposed system to a realistic scenario, the peak delivery demand peak is effectively leveled. 
Number of deliveries needed in as is and proposed system

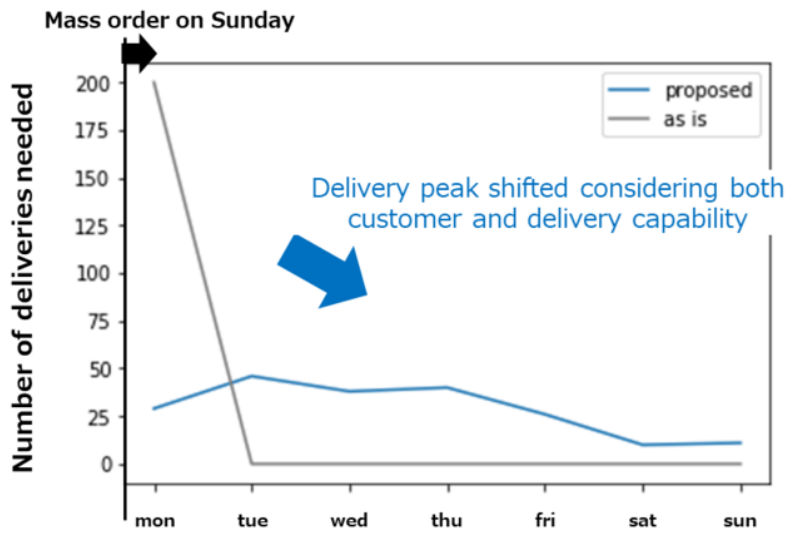

Figure 8. Number of deliveries needed in as-is and proposed system.

\section{Conclusion}

In this paper, an alternative transdisciplinary approach for demand peak leveling was investigated and proposed. From the customer acceptance capability analysis and delivery capablitliy analysis, the possiblity of delivery re-allocation without lowering or even uplifting customer utility, was shown. Based on this implication, a realtime delivery allocation system was propose, and throught a case study, its effect was evaluated and verified.

Future works could be

1. constructing an algorythm to estimate customer acceptance capabitliy for customers with small or no data

2. making the devliery capacity analysis finer by analyzing and modeling each destination's off-truck time

3. inserting an delivery re-allocation incentive process in the system to make the allocation judgement more decentralized

By combining conventional optimization approaches and the proposed delivery allocation approach, a social-optimal supply chain could be constructed.

\section{References}

[1] Yano Research Institute, 2019, Yano Research Institute, Accessed: 2002 2020. [Online]. Available: https://www.yanoresearch.com/en/press-release/show/press_id/2183

[2] N.A. El-Sherbeny, Vehicle routing with time windows: An overview of exact, heuristic and metaheuristic methods, Journal of King Saud University-Science, 2010, 22.3, pp. 123-131.

[3] V. Sanchez Rodrigues, A. Potter, M.M. Naim, Evaluating the causes of uncertainty in logistics operations, The International Journal of Logistics Management, 2010, Vol. 21, pp. 45-64.

[4] X.L. Wang, S.-H. Ma, Research on Capacity Coordination in a Logistics Service Supply Chain With Demand and Supply Uncertainties, Operations Research and Management Science, 2011, 2, pp. 44-49.

[5] U.S. Department of Energy, Benefits of Demand Response in Electricity Markets and Recommendations for Achieving Them, 2006. 